\title{
Inversión pública y pobreza: el caso ecuatoriano hasta 2020
}

Public investment and poverty: the ecuadorian case up to 2020

Ismael Bravo Placeres

Fecha de recepción: 22 de abril de 2019

Fecha de aceptación: 10 de junio de 2019 


\title{
Inversión pública y pobreza: el caso ecuatoriano hasta 2020
}

\author{
Public investment and poverty: the ecuadorian case up to 2020
}

Ismael Bravo Placeres ${ }^{1}$

Como citar: Bravo, I., (2019). Inversión pública y pobreza: el caso ecuatoriano hasta 2020, Revista Universidad de Guayaquil. 129(2), 38-55. DOI: https://doi.org/10.53591/rug.v129i2.1376

\section{RESUMEN}

El presente artículo examina la incidencia de la inversión pública en el Ecuador. El análisis de la problemática considera la situación de la pobreza en el país, un flagelo de proporciones crecientes a escala global, especialmente en los últimos años. El análisis parte de la literatura oficialmente publicada por el gobierno ecuatoriano y el procesamiento de fuentes secundarias. La metodología empleada en el trabajo es mixta, con soporte en los métodos propios de la metodología cualitativa para el análisis documental de obras, ensayos y artículos referidos a los distintos enfoques y teorías sobre la inversión pública y la pobreza. Los datos utilizados son de origen secundario, pues la información estadística proviene de instituciones como el Instituto Nacional de Estadística y Censo (INEC), Secretaria Nacional de Planificación y Desarrollo (SENPLADES), Sistema Integrado de Conocimiento y Estadística Social del Ecuador (SiCES), Plan Nacional para el Buen Vivir (PNBV), entre otros.

Palabras clave: Pobreza, Inversión Pública, Políticas Públicas, Bienestar.

\section{ABSTRACT}

The present article examines the incidence of the public investment in the Ecuador. The analysis of the problem considers the situation of the poverty in the country, an I lash from growing proportions to global scale, especially in the last years. The analysis leaves of the literature officially published by the Ecuadorian government and the prosecution of secondary sources. The methodology used in the work is mixed, with support in the methods characteristic of the qualitative methodology for the documental analysis of works, rehearsals and articles referred to the different focuses and theories about the public investment and the poverty. The used data they are of secondary origin, because the statistical information comes from institutions like the National Institute of Statistic and Census (INEC), National Secretary of Planning and Development (SENPLADES), Integrated System of Knowledge and Social Statistic of the Ecuador (SiCES), National Plan for the Good one to Live (PNBV), among others.

Keywords: Poverty, Public Public, Political Investment, Well-being.

\footnotetext{
${ }^{1}$ Máster en Gestión Turística. Docente de la Carrera de Turismo, Facultad de Ciencias Administrativas, Universidad de Guayaquil. Ecuador.
} Correoelectrónico: ismel.bravopla@ug.edu.ec 


\section{INTRODUCCIÓN}

La pobreza se ha convertido en una verdadera pandemia que azota a la humanidad sin distinción de países y continentes. El impacto es mucho mayor en algunas regiones del planeta, donde millones de personas, no solo son pobres, sino que viven en condiciones de extrema pobreza, y son víctimas de enfermedades curables, que incluso ya han desaparecido en países desarrollados.

El tema de la pobreza ha sido tratado por múltiples especialistas, según (Bazán Ojeda \& Quintero Soto, 2011) es definida como la cualidad de pobre, haciendo referencia a las personas que no tienen lo necesario para vivir dignamente, que son humildes o que son desdichadas. La Organización de Naciones Unidas definió la pobreza como "la condición caracterizada por una privación severa de necesidades humanas básicas incluyendo alimentos, agua potable, instalaciones sanitarias, salud, vivienda, educación e información. La pobreza depende no sólo de ingresos monetarios, sino también del acceso a servicios"(ONU, 1995).

La pobreza va arraigada y sujeta a la falta de uno u otro renglón socio-económico, a saber: salud, educación, vivienda, ingresos, empleo, agricultura estable, nutrición, tecnología, mortalidad infantil, enfermedades curables. Algunas de estas cualidades son tangibles, o sea, se pueden medir; y otras que son intangibles, por ello se puede afirmar que tiene características tangibles e intangibles. Las primeras se refieren a algunos aspectos (y su calidad) imprescindibles para la vida, entre los que se encuentran: la vivienda, salud, empleo, alimentación, educación y el nivel de ingresos. En el caso de los intangibles, se suelen considerar: derechos humanos, género, identidad cultural, participación ciudadana, información y legalidad (PNUD, 2015)

Como se observa, el análisis de la pobreza resulta altamente complejo debido a la naturaleza multidimensional de la misma, que generalmente es enfocada desde una perspectiva solamente económica, y reduce los impactos políticos sociológicos, ideológicos y medioambientales de la misma. Las cifras publicadas por el Banco Mundial (Banco Mundial, 2020) demuestran que hasta ese año la tasa de pobreza extrema mundial disminuyó del 10,1\% en 2015 al 9,2\% en 2017, lo que equivale a 689 millones de personas que vivían con menos de USD 1,90 al día. En 2017, en las líneas de pobreza más altas, el 24,1 \% del mundo vivía con menos de USD 3,20 al día y el 43,6 \% con menos de USD 5,50. Un reporte especializado de esta fuente refleja que:

- En 2018, de cada cinco personas por debajo de la línea internacional de pobreza, cuatro vivían en zonas rurales. 
- La mitad de los pobres son niños. Las mujeres representan la mayoría de los pobres en la mayor parte de las regiones y en algunos grupos de edad. De la población mundial pobre de 15 años o más, alrededor del $70 \%$ no tiene ninguna formación o solo una instrucción básica.

- Casi la mitad de los pobres de África al sur del Sahara viven en solo cinco países: Nigeria, República Democrática del Congo, Tanzanía, Etiopía y Madagascar.

- Más del $40 \%$ de los pobres del mundo vive en economías afectadas por la fragilidad, los conflictos y la violencia, y se espera que esa cifra aumente al $67 \%$ en la próxima década. Esas economías representan el $10 \%$ de la población mundial.

- Alrededor de 123 millones de personas pobres en el mundo viven en zonas con alto riesgo de inundaciones.

En este contexto, América Latina continúa siendo una de las regiones con mayor desigualdad del mundo, y la pobreza juega un papel principal en esta situación. Mientras que en algunos países la mayoría o una minoría muy amplia de la población vive en algún nivel de pobreza, en otros esta región la pobreza se encuentra prácticamente erradicada o en unas cifras muy limitadas (EOM, 2019) (Figura 1)

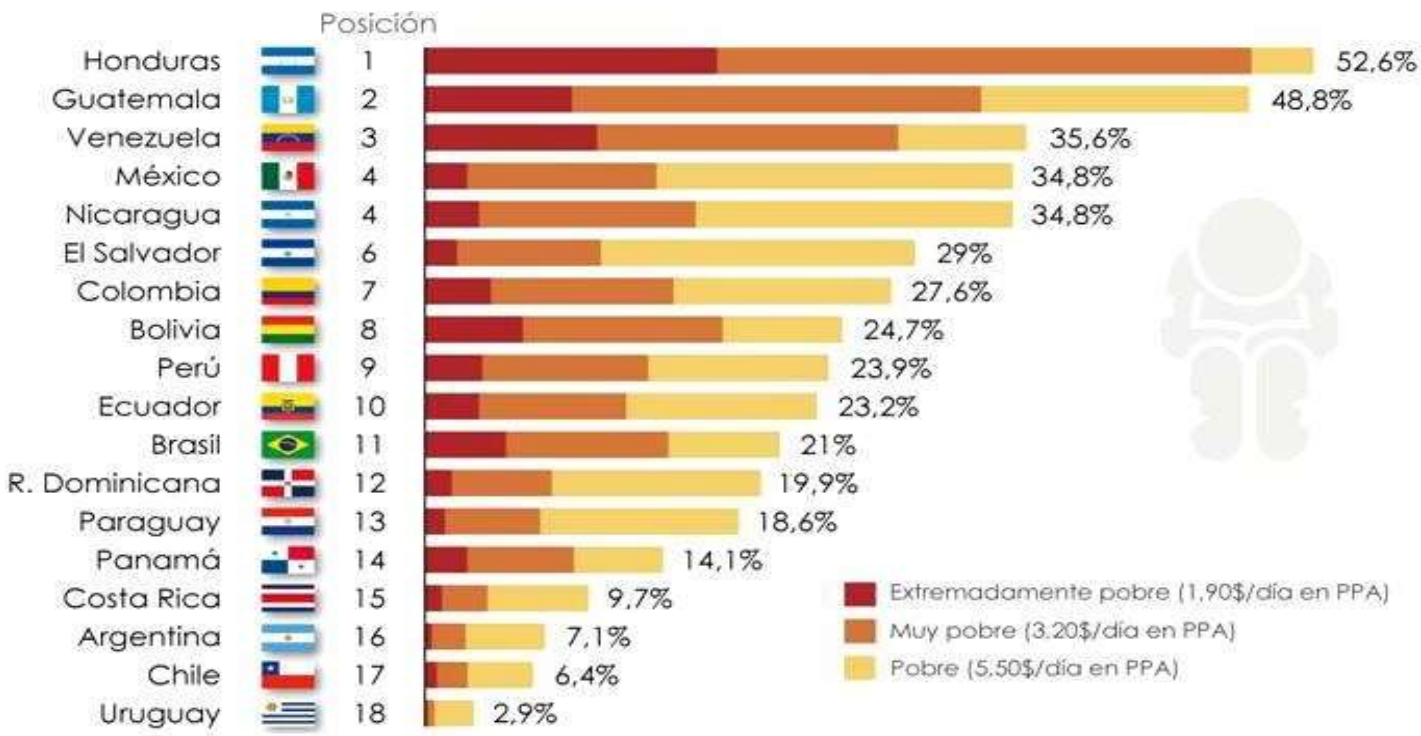

Figura 1. Gráfico que muestra un análisis porcentual de la población que vive en la pobreza en los países de América Latina según reportes del Banco Mundial. Fuente: EOM, 2019a.

En el orden práctico, los gobiernos e instituciones se enfrentan al problema de determinar cuál es el nivel mínimo de vida para que una persona ya no sea considerada pobre, así como poder realizar comparaciones entre personas que viven en distintos territorios para conocer cuáles son 
los gastos necesarios en circunstancias específicas para asegurar el nivel de vida mínimo por encima de la pobreza. Una tentativa de solución está en la determinación de las líneas de pobreza (Ravallion, 2015). Internacionalmente se utilizan varios métodos para medir la situación de pobreza: la pobreza absoluta cuando ciertos estándares mínimos de vida, tales como: la nutrición, salud y vivienda no pueden ser alcanzados (Girondella Mora, 2011), (León Segura, 2016). Pobreza relativa cuando no se tiene el nivel de ingresos necesarios para satisfacer todas o parte de las necesidades básicas de acuerdo a los criterios de un determinado tiempo y sociedad (Niemietz, A new understanding of poverty, 2011).

El estudio del problema de la pobreza ha transitado por diferentes enfoques, que además de analizar sus relaciones de causalidad, consecuencias y diferentes formas de medición, son la base de la formulación de diferentes políticas públicas para su erradicación. Entre ellos, el enfoque de la pobreza en función de la relación ingreso-consumo, a pesar de que no hay suficiente evidencia empírica sobre la correlación entre los niveles de ingreso y de bienestar (Hume, Understanding and explaining global proverty. Global proverty : how global governanve is failing the poor.Londres: Routledge, 2012).

A partir de este enfoque directo se utiliza como forma de medición: El método indirecto (o método del ingreso o consumo), mide el nivel de vida a partir de los ingresos y consumo de las personas u hogares. Parte de la estimación de una línea de pobreza, definida como el costo mínimo de una canasta de bienes y servicios que satisfaga las necesidades básicas (alimentación, vivienda, vestido, educación y salud).

Otra forma de medir la pobreza es utilizando el método de las necesidades básicas insatisfechas (NBI) o de los indicadores sociales) a partir de la tesis de la CEPAL que define un hogar como pobre cuando adolece de carencias graves en el acceso a la educación, la salud, la nutrición, la vivienda, servicios urbanos y oportunidades de empleo (Comisión económica para América Latina y el Caribe, 2006)

Particularmente en la República del Ecuador, la disminución de los niveles de pobreza se ha logrado a través del diseño y aplicación de políticas públicas direccionadas en cinco ejes fundamentales: Bienestar social, educación, salud, trabajo, vivienda y desarrollo urbano. La medición de la pobreza en esta nación se realiza utilizando el índice de pobreza multidimensional, aplicado desde diciembre del 2015, el cual fue construido teniendo en cuenta los resultados de la encuesta urbana de empleo y desempleo del período 2005-2012, y fue elaborado a partir de la metodología de Alkire y Lester, diseñada por la Universidad de Oxford. 
A partir del análisis de los derechos constitucionales establecidos en Ecuador y sus respectivos referentes socios-económicos institucionales, se realiza en el desarrollo del trabajo, un análisis crítico de la bibliografía referida al tema de la pobreza en Ecuador, así como de las estadísticas oficiales publicadas por instituciones ecuatorianas.

\section{MATERIALES Y MÉTODOS}

La investigación presentada es de tipo mixta, es decir, se combinan elementos cualitativos para el estudio de las teorías y enfoques relativos a la pobreza, la inversión y el gasto público en el sector salud. Los datos estadísticos se recolectan a partir de las fuentes oficiales del gobierno de Ecuador y se utilizan métodos de correlación para el análisis de la relación entre las variables definidas en su entorno natural.

Desde el punto de vista del alcance de la investigación podemos decir que es de tipo descriptiva, pues se procede a la caracterización del marco legal y la metodología para la determinación de la pobreza, las líneas de pobreza y su métrica a través del Índice de Pobreza Multidiensional. Por último, se describe la relación entre el gasto y la inversión pública en el Producto Interno Bruto (PIB), así como la incidencia de esta en los niveles de pobreza en el período objeto de estudio.

\section{RESULTADOS Y DISCUSIÓN}

La Constitución del Ecuador (2008) y el Plan Nacional del Buen Vivir (2013) delinearon el marco normativo, la estrategia de desarrollo y los objetivos a alcanzar para disminuir los niveles de pobreza en este país. La Constitución de Ecuador del 2008 elevó por primera vez la noción del Buen Vivir como directriz fundamental del Estado, estableciéndose como derecho de los individuos y las distintas colectividades a la hora de construir una sociedad mejor, entiéndase, plural, justa, incluyente, en armonía con la naturaleza. Según Galiano y Tamayo (2018), esta novedosa cosmovisión de origen indígena cristalizó en un principio constitucional de cardinal importancia, el Buen vivir o Sumak Kawsay, el cual trajo consigo el reconocimiento de una serie de derechos y garantías de índole social, económica, ambiental y política. Así, el buen vivir comprende los derechos reglamentados en el capítulo II del título II del texto constitucional, y en el régimen establecido en el título VII, relativo al Plan Nacional de Desarrollo 2017-2021 “Toda una vida” (Galiano Maritan \& Tamayo Santana, 2018).

En la Constitución actual del Ecuador, el Buen Vivir se encuentra relacionado con una serie de derechos que se regulan en el ordenamiento constitucional ecuatoriano vigente, donde la 
conceptualización del Buen Vivir es el paradigma del modelo y la política de desarrollo del país.

El Plan Nacional del Buen Vivir (2017-2021) asume que esta es la forma de vida que permite la felicidad y la permanencia de la diversidad cultural y ambiental; es armonía, igualdad, equidad y solidaridad. No es buscar la opulencia ni el crecimiento económico infinito. Los objetivos 2, 3, 4, y 9 también trazan las directrices para la elaboración de políticas públicas encaminadas a combatir la pobreza (Secretaría Nacional de Planificación y Desarrollo Senplades, 2017).

La noción del Buen Vivir en Ecuador está impregnada en toda la Constitución, tal es así que aparece desde el preámbulo de la constitución, cuando se establece que el pueblo soberano de Ecuador construirá una nueva forma de convivencia ciudadana, en diversidad y armonía con la naturaleza, para alcanzar el buen vivir como paradigma de igualdad, el reconocimiento de derechos y la satisfacción plena de las necesidades tanto objetivas como subjetivas de las personas y los pueblos. Noventa y Nueve son los artículos de la Constitución del Ecuador en los cuales se hace referencia expresa al Buen Vivir, Así, el capítulo II, del título II reconoce los derechos del Buen Vivir; en el título VI se establece el régimen de desarrollo (conjunto organizado, sostenible y dinámico de los sistemas económicos, políticos, socio-culturales y ambientales), y en el título VII, se establece el régimen del Buen Vivir. De allí, el artículo 3 de la Constitución establece que constituye un deber del Estado: "Planificar el desarrollo nacional, erradicar la pobreza, promover el desarrollo sustentable y la redistribución equitativa de los recursos y la riqueza, para acceder al buen vivir" (Constitución de la República del Ecuador, 2008).

Por ello, dentro del texto constitucional, constituyen derechos del Buen Vivir relacionados específicamente a la erradicación de la pobreza los siguientes: 1. Agua y alimentación (arts. 12 y 13); 2. Ambiente sano (arts. 14 y 15); 3. Comunicación e información (arts. 16-20); 4. Cultura y ciencia (arts. 21-25); 5. Educación (arts. 26-29); 6. Hábitat y vivienda (arts. 30 y 31); 7. Salud (art. 32); 8. Trabajo y seguridad social (arts. 33 y 34). Se regula además en los artículos 340 al 394, la inclusión y equidad (educación, salud, seguridad social, hábitat y vivienda, cultura, cultura física y tiempo libre, comunicación social, ciencia, tecnología, innovación y saberes ancestrales, gestión del riesgo, población y movilidad humana, seguridad humana y transporte); y las atinentes a la biodiversidad y los recursos naturales (naturaleza y ambiente, biodiversidad, patrimonio natural y ecosistemas, recursos naturales, suelo, agua, biosfera, ecología urbana y energías alternativas) en los artículos 395 a 415. 
El Ciclo de Política de Inversión Pública Nacional en Ecuador inicia con la formulación del Plan Nacional de Desarrollo, donde a través de un proceso participativo se definen los objetivos estratégicos y se proyecta la Inversión Plurianual (4 años) alineada a cada uno de los objetivos del plan.

En Ecuador las estadísticas oficiales sobre la inversión pública, estado de la pobreza y otros renglones, son publicadas por el Ministerio Coordinador de Desarrollo Social en su llamado "Sistema integrado de Conocimiento y Estadística Social del Ecuador" (en lo adelante SiCES), el cual emplea diversos estudios realizados por las instituciones de este país, mostrando un decrecimiento significativo de la pobreza entre los años 2000 y 2017 (Figura 2) que estuvo acompañado de un significativo incremento de las inversiones públicas en la nación (Figura 3).

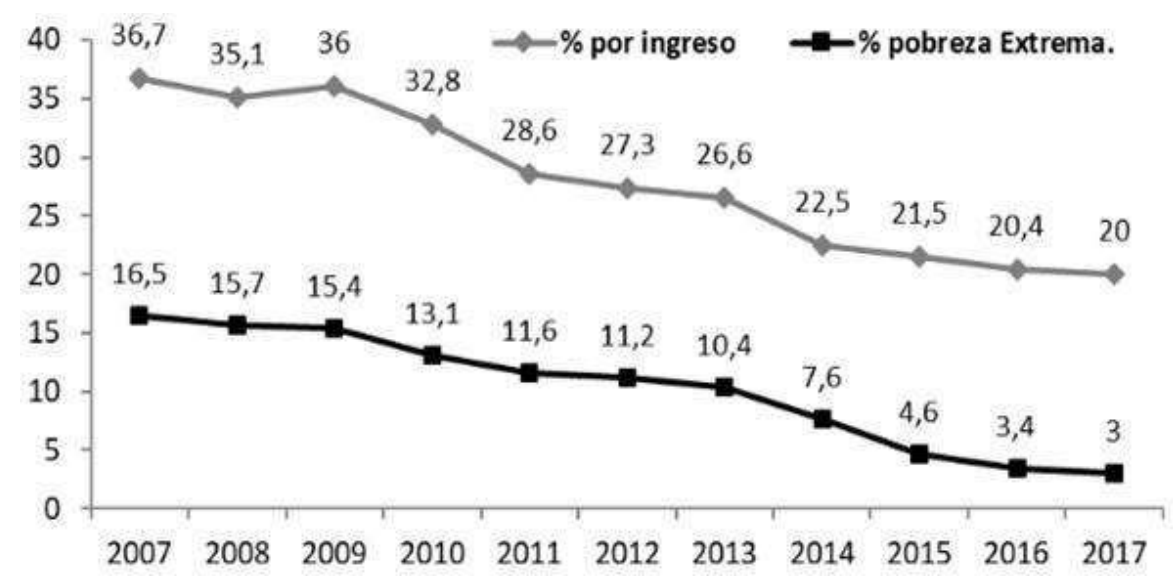

Figura 2. Porcentaje de Pobreza en el Ecuador. Fuente: Mero y Alvarado, 2018 (Mero Figueroa \& Alvarado-Gastiaburo, 2018)

Pol. Con. (Edición núm. 24) Vol. 3, No Esp. 1

Septiembre 2018, pp. 458-485 ISSN: 2550 - 682X

DOI: 10.23857/pc.v3i1 Esp.695 


\section{Tendencias de la pobreza y la desigualdad en el Ecuador y América Latina}

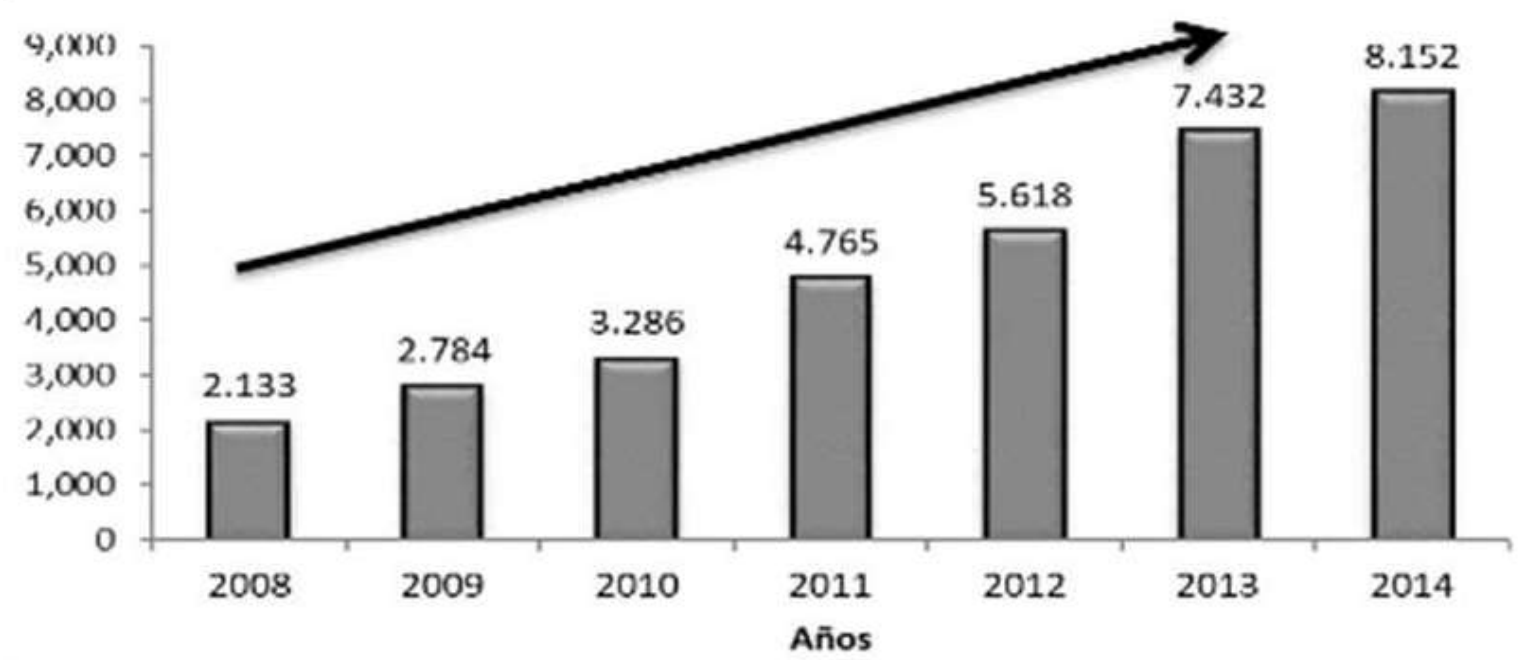

Figura 3. Gráfico que muestra el crecimiento de la Inversión pública en el Ecuador, expresada en millones de dólares. Fuente: Mero y Alvarado, 2018.

Según las estadísticas publicadas por SiCES en su página web, uno de los sectores en los cuales las políticas públicas han incidido más favorablemente en la disminución de la pobreza en Ecuador es el sector de la Educación. (Tabla 1)

Tabla 1. Evolución de la inversión pública en el producto interno bruto (PIB) ecuatoriano en el período 2014-2017. Fuente: SiCES. (2020)

\begin{tabular}{cc}
\hline Años & Inversión pública en Educación \\
\hline $\mathbf{2 0 1 4}$ & $4,70 \%$ \\
$\mathbf{2 0 1 5}$ & $4,50 \%$ \\
$\mathbf{2 0 1 6}$ & $4,40 \%$ \\
$\mathbf{2 0 1 7}$ & $4,70 \%$ \\
\hline
\end{tabular}

La información disponible muestra que que la inversión pública en Ecuador en el sector Educación tiene una incidencia alta como porcentaje del PIB, ha tenido una variación significativa en los años 2015 y 2016, para el 2014 representaba el 4,70\% de la inversión total, en el 2015y 2016 disminuyó entre en 20\% y 30\% debido a la caída de los precios del petróleo y el terremoto que azotó las provincias de Manabí y Esmeraldas en abril del 2016. Los montos de inversión pública en el sector de salud también han sido significativos como se detalla en la tabla 2 . 
Tabla 1. Inversión Pública como porcentaje del PIB en la salud 2014 - 2017. Fuente: SiCES. (2020)

\begin{tabular}{lc}
\hline Años & Inversión Pública en Salud \\
\hline $\mathbf{2 0 1 4}$ & $2,2 \%$ \\
$\mathbf{2 0 1 5}$ & $2,8 \%$ \\
$\mathbf{2 0 1 6}$ & $2,9 \%$ \\
$\mathbf{2 0 1 7}$ & $3,2 \%$ \\
\hline
\end{tabular}

La inversión como porcentaje en el PIB ha sido creciente en el período 2014 - 2017, ya que ha tenido un aumento de un punto porcentual en cada año.

La vivienda y el desarrollo urbano tienen un alto impacto en la disminución de la pobreza y el aumento del bienestar y la calidad de la vida. La intervención del sector de la vivienda y desarrollo urbano como porcentaje del PIB, ha sido cíclica ya que ha habido aumento y disminución, debido a que en el 2014 estaba representado por el 0,8\%, la cual es la más alta de los últimos 4 años, para el 2015 se tuvo una diminución porcentual del 0,7\%, alcanzando el 0,1\% de participación en ese año por problema económicos en el país, en el 2016 se elevó a 0,3\% y luego para el 2017 nuevamente tuvo un decrecimiento llegando al 0,2\% (SiCES, 2020), llegando a más de 255.000 viviendas entregadas en seis años (Miniterio de Desarrollo Urbano, 2020) (Tabla 3) (Tabla 4) (Figura 4)

Tabla 2: Inversión Pública como porcentaje del PIB en vivienda y desarrollo urbano 2014 2017. Fuente: SiCES. (2020)

\begin{tabular}{lc}
\hline Años & $\begin{array}{c}\text { Inversión Pública en Vivienda y } \\
\text { Desarrollo Urbano }\end{array}$ \\
\hline $\mathbf{2 0 1 4}$ & $0,8 \%$ \\
$\mathbf{2 0 1 5}$ & $0,1 \%$ \\
$\mathbf{2 0 1 6}$ & $0,3 \%$ \\
$\mathbf{2 0 1 7}$ & $0,2 \%$ \\
\hline
\end{tabular}


Tabla 4: Inversión Pública como porcentaje del PIB en trabajo 2014 - 2017. Fuente: SiCES. (2020)

\begin{tabular}{lc}
\hline Años & Inversión Pública en Trabajo \\
\hline $\mathbf{2 0 1 4}$ & $0,11 \%$ \\
$\mathbf{2 0 1 5}$ & $0,05 \%$ \\
$\mathbf{2 0 1 6}$ & $0,05 \%$ \\
$\mathbf{2 0 1 7}$ & $0,05 \%$ \\
\hline
\end{tabular}
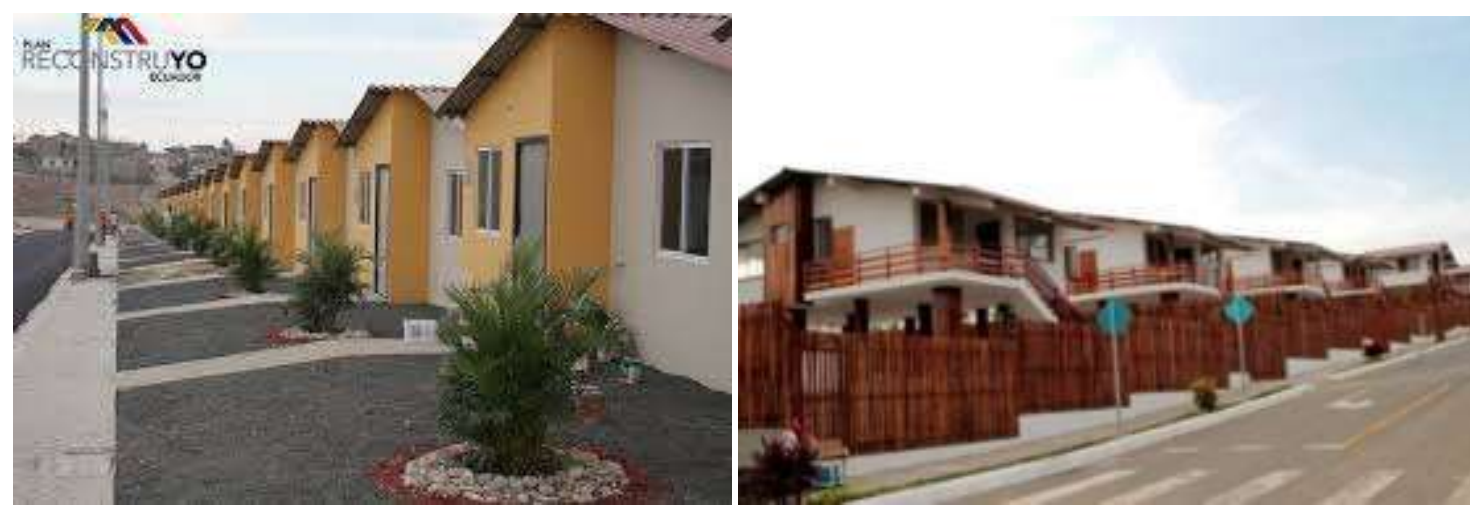

Figura 4. Imagen de viviendas entregadas por el gobierno en Manabí del programa Reconstruyo en 2018 (Izquierda) Fuente: https://www.reconstruyoecuador.gob.ec/91viviendas-se-entregaron-el-miercoles-en-manabi/ . Comunidad del Milenio "Ciudad Jardín”, ubicada en Tablada de Sánchez, cerca de la zona de Río Grande en el Cantón Chone. (Derecha) Fuente: https://www.eltelegrafo.com.ec/noticias/politica/2/inaugurado-plan-habitacional-quecomprende-81-viviendas

La inversión pública direccionada a la creación de puestos de trabajo presentó una disminución porcentual en el 2014, manteniéndose desde entonces sin variación, siendo la generación de empleos uno de los problemas más acuciantes que debe enfrentar la economía del país para mantener los logros alcanzados en la disminución de la pobreza y elevar el bienestar y calidad de vida de la población. (Tabla 5) 
Tabla 5: Índice de pobreza multidimensional en Ecuador al cierre del 2017. Fuente: SiCES. (2020)

\begin{tabular}{llll}
\hline Años & \multicolumn{3}{c}{ IPM } \\
\cline { 2 - 4 } & Nacional & Urbana & Rural \\
\hline $\mathbf{2 0 1 4}$ & $18,1 \%$ & $10,7 \%$ & $33,8 \%$ \\
$\mathbf{2 0 1 5}$ & $17,0 \%$ & $9,3 \%$ & $33,4 \%$ \\
$\mathbf{2 0 1 6}$ & $16,9 \%$ & $10,2 \%$ & $31,2 \%$ \\
$\mathbf{2 0 1 7}$ & $16,9 \%$ & $9,1 \%$ & $33,6 \%$ \\
\hline
\end{tabular}

El reporte de pobreza y desigualdad de junio 2018 del Ecuador, registra que la pobreza por ingresos a nivel nacional en junio de 2018 se mantiene constante estadísticamente y se ubicó en un $24,5 \%$. Para el mismo período, la pobreza tanto a nivel rural (43,0\%), como a nivel urbano $(15,9 \%)$, no presentan variaciones estadísticamente significativas (INEC, 2018).

El coeficiente de Gini se ubicó en junio de 2018 en 0,472 a nivel nacional, 0,452 en el área urbana, y 0,448 en el área rural. Las variaciones respecto al año anterior no fueron estadísticamente significativas (INEC, 2018).

En relación a la línea de pobreza y pobreza extrema se realizan en base a los estándares internacionales: El Instituto Nacional de Estadísticas de Ecuador -INEC utiliza recomendaciones internacionales, para lo cual se compara el ingreso per cápita familiar con la línea de pobreza y pobreza extrema, que en el mes de junio de 2018 se ubicaron en $\$ 84,72$ y \$ 47,74 mensuales por persona respectivamente. Los individuos cuyo ingreso per cápita es menor a la línea de pobreza son considerados pobres, y si es menor a la línea de pobreza extrema son considerados pobres extremos" (INEC, 2018). (Tabla 6)

Tabla 6: Coeficiente de Gini en Ecuador 2014 - 2017. Fuente: SiCES. (2020)

\begin{tabular}{llll}
\hline \multirow{2}{*}{ Años } & \multicolumn{3}{c}{ Coeficiente de Gini } \\
\cline { 2 - 4 } & Nacional & Urbano & Rural \\
\hline $\mathbf{2 0 1 4}$ & $0,49 \%$ & $0,47 \%$ & $0,44 \%$ \\
$\mathbf{2 0 1 5}$ & $0,48 \%$ & $0,45 \%$ & $0,48 \%$ \\
$\mathbf{2 0 1 6}$ & $0,47 \%$ & $0,45 \%$ & $0,46 \%$ \\
$\mathbf{2 0 1 7}$ & $0,46 \%$ & $0,44 \%$ & $0,46 \%$ \\
\hline
\end{tabular}

El índice de Gini mide el grado de desigualdad comprendida entre cero y está medido a través del ingreso per cápita, para el año 2014 este coeficiente a nivel nacional se centró 0,49 la urbana 
en $0,47 \%$ y la rural en $0,44 \%$ existiendo una variación en los años posteriores terminando en el 2017 con un coeficiente a nivel nacional de $0,46 \%$ en la zona urbana $0.44 \%$ y en la rural en 0,46\% este se ha mantenido con respecto al año 2016 mostrando que la desigualdad aún se encuentra adyacente en el sector rural.

La amenaza más reciente e inmediata para la reducción de la pobreza, la pandemia de COVID19 (coronavirus), ha desencadenado un desastre económico mundial cuya onda expansiva sigue propagándose. Sin una respuesta mundial adecuada, los efectos acumulativos de la pandemia y sus repercusiones económicas, de los conflictos armados y del cambio climático se cobrarán un alto costo humano y económico hasta bien avanzado el futuro. Las últimas investigaciones sugieren que, casi con toda seguridad, los efectos de la actual crisis se harán sentir en la mayoría de los países hasta 2030. En estas condiciones, el objetivo de reducir la tasa absoluta mundial de pobreza a menos del $3 \%$ para 2030 — que ya se encontraba comprometido antes de la crisis - es ahora inalcanzable si no se adoptan medidas políticas rápidas, significativas y sustanciales (Banco Mundial, 2020). A pesar de todo, muchas de las personas que apenas habían escapado de la pobreza extrema podrían recaer en ella como consecuencia de la convergencia de la pandemia de COVID-19, los conflictos y el cambio climático. Según un "pronóstico inmediato" (estimación preliminar) sobre 2020 en el que se incorporan los efectos de la pandemia de COVID-19, se calcula que esta empujará a entre 88 millones y 115 millones más de personas a la pobreza extrema, con lo que el total se situará entre 703 millones y 729 millones (Banco Mundial, 2020).

Según OXFAM Internacional (2021), en tan solo nueve meses, las mil mayores fortunas del mundo ya habían recuperado las pérdidas económicas originadas por la pandemia de COVID 19, mientras que las personas en mayor situación de pobreza podrían necesitar más de una década para recuperarse de los impactos económicos de la crisis. Esta fuente muestra cómo nuestro sistema económico fallido permite que una élite súper rica continúe acumulando riqueza en medio de la mayor crisis económica desde la Gran Depresión, mientras miles de millones de personas se enfrentan a grandes dificultades para salir adelante. Según expresa Annie Thériault (Especialista de OXFAM Internacional), el informe también revela cómo la pandemia está profundizando las históricas desigualdades económicas, raciales y de género (OXFAM Internacional, 2021):

- La recesión ya ha acabado para los más ricos. Desde el inicio de la pandemia, la fortuna de los 10 hombres más ricos del mundo ha aumentado en medio billón de dólares, una cifra que financiaría con creces una vacuna universal para la COVID-19 y que 
garantizaría que nadie cayese en la pobreza como resultado de la pandemia. Al mismo tiempo, la pandemia ha desencadenado la peor crisis laboral en más de 90 años, y cientos de millones de personas se encuentran subempleadas o sin trabajo.

- De nuevo, las mujeres son las más afectadas. A nivel mundial, las mujeres están sobrerrepresentadas en trabajos mal remunerados y precarios, que han sido los que más se han visto afectados por la crisis de COVID-19. Si la presencia de hombres y mujeres en dichos sectores fuese totalmente equitativa, 112 millones de mujeres dejarían de tener un riesgo elevado de perder sus ingresos o empleos. Al mismo tiempo, las mujeres constituyen aproximadamente el $70 \%$ de la fuerza laboral a nivel mundial en el ámbito de la salud y la atención social, empleos esenciales, pero a menudo mal remunerados que además las exponen a un mayor riesgo de contraer el virus.

- La desigualdad se está cobrando vidas. En Brasil, las personas afrodescendientes tienen un $40 \%$ más de probabilidades de morir a causa de la COVID-19 que las personas blancas, mientras que, en los Estados Unidos, si la tasa de mortalidad de las personas de origen latino y afroamericano hubiese sido la misma que la de las personas blancas, aproximadamente 22000 personas negras y latinas aún seguirían con vida. Las zonas más pobres de países como España, Francia e India presentan tasas de infección y mortalidad más elevadas. En el caso de Inglaterra, las tasas de mortalidad de las regiones más pobres duplican a las de las zonas más ricas.

- La clave para lograr una rápida recuperación económica frente a la pandemia es la adopción de modelos económicos más justos. La aplicación de un impuesto temporal sobre los beneficios excesivos obtenidos por las 32 multinacionales que mayor riqueza han acumulado desde que comenzara la pandemia habría permitido recaudar 104000 millones de dólares en 2020, cantidad suficiente para financiar prestaciones por desempleo para todos los trabajadores y trabajadoras, así como para proporcionar apoyo económico a todos los niños, niñas y personas mayores de los países de renta media y baja.

Ecuador no ha estado exento del impacto de la COVID-19, causando incrementos en el nivel pobreza. Según la página digital Primicias.ec (2021), la actual crisis por la pandemia de Covid19 impacta especialmente a las mujeres y a los grupos étnicos y en situación de exclusión, porque son grupos que tienen mayores probabilidades de verse arrastrados a la pobreza, de pasar hambre y no tener acceso a servicios de salud. En el mundo, las mujeres están 
sobrerrepresentadas en trabajos mal remunerados y precarios, que han sido los más golpeados por la crisis. En Ecuador uno de esos empleos es el de las trabajadoras remuneradas del hogar que, durante la pandemia, han experimentado despidos, mayor carga laboral y exposición al Covid-19. "Si la presencia de hombres y mujeres en todas las actividades económicas fuese totalmente equitativa, 112 millones de mujeres dejarían de tener un riesgo elevado de perder sus ingresos o empleos. A eso se suma el hecho de que las mujeres representan aproximadamente el $70 \%$ de la fuerza laboral mundial en el ámbito de la salud y la atención social. Esos empleos, que son considerados esenciales, exponen a las mujeres a bajas remuneraciones y a un mayor riesgo de contraer el virus (Primicias.ec, 2021).

Según OXFAM Internacional (2021), los grupos que suelen tener empleos más precarios y trabajar en el sector informal también tienen más probabilidades de que su situación laboral y sus ingresos se hayan visto más afectados por la pandemia (Figura 5).

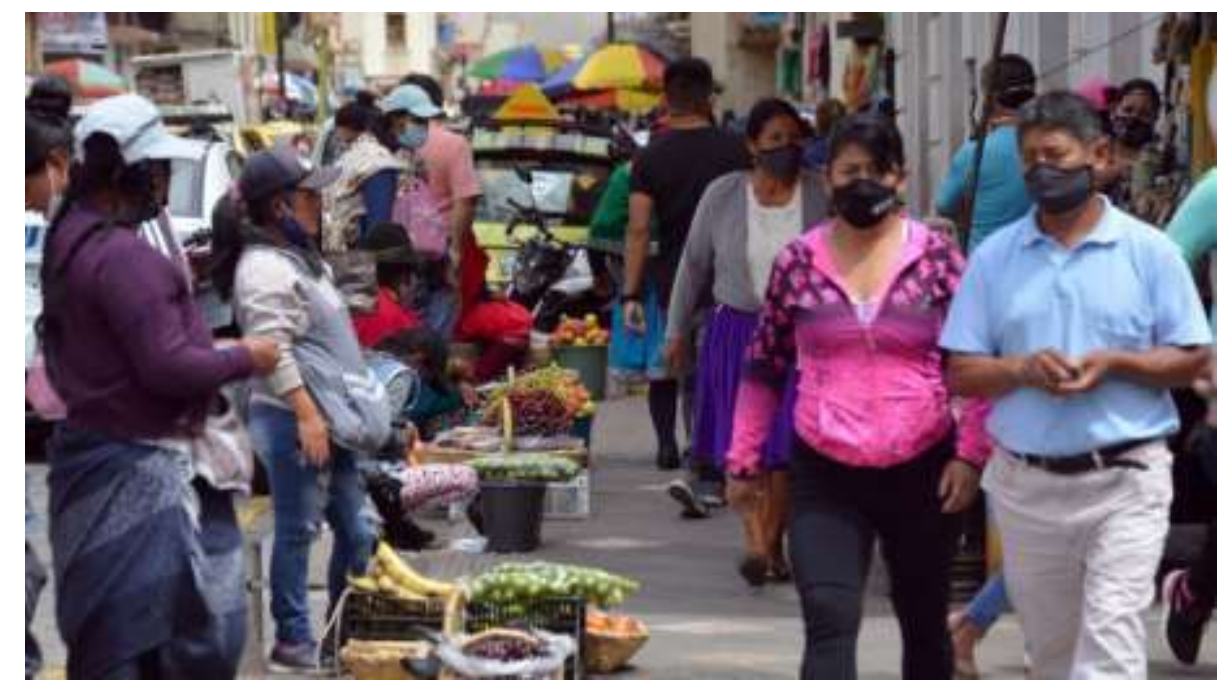

Figura 5. Personas el sector informal venden frutas en una calle de Cuenca, Ecuador, en enero de 2021. Fuente: https://www.primicias.ec/noticias/economia/pobres-necesitan-decada-pararecuperarse-ricos-ya-hicieron-covid/

Los datos expresados en el informe de OXFAM Internacional (2021), indican que, en América Latina, las personas afrodescendientes y los pueblos indígenas están sufriendo en mayor medida la pobreza provocada por la pandemia. Específicamente son grupos que tienen más probabilidades de no tener ingresos para satisfacer sus necesidades básicas y de morir a causa del virus. No solo América Latina experimenta la desigualdad racial, países como Estados Unidos, Reino Unido, Francia, España e India también lo hacen. En Estados Unidos el índice de pobreza de la población blanca se incrementará en 4,2 puntos porcentuales, de la población 
latina en 9,4 puntos porcentuales y de la población negra en 12,6 puntos porcentuales (Primicias.ec, 2021).

Como puede observarse, en el caso del Ecuador, los aciertos en la reducción de los niveles de pobreza gracias a las políticas públicas aplicadas por los gobiernos en los últimos 15 años anteriores al 2020, hoy se ven amenazados por la situación de la pandemia de COVID-19. Quedan muchos retos, pero dependerá de la voluntad gubernamental y, particularmente del trabajo colectivo de todos los actores sociales, luchar por lograr el sueño de la política del Buen Vivir.

\section{CONCLUSIONES}

1. De los montos de los rubros de la inversión pública en el período 2014-2017, ha sido en la educación y la vivienda los sectores que más se invierte, garantizando el acceso y la calidad de la enseñanza en todas sus fases, y nuevas viviendas dignas, con una participación del $4.70 \%$ del PIB, lo cual es muestra de una voluntad direccionada al desarrollo social, tomando en cuenta los shocks que atravesó la economía ecuatoriana durante los años 2015-2016, en que todos los rubros de la inversión pública bajaron por la crisis de la caída de los precios del petróleo y el terremoto en la provincia de Esmeraldas.

2. Estadísticamente, no hay variaciones significativas en relación a la pobreza urbana y rural, y el coeficiente de GINI muestra una pequeña disminución. Han incidido en estos resultados las políticas públicas dirigidas a sectores como educación, salud e infraestructura. En tanto, los resultados de la inversión pública en generación de empleo y desarrollo urbano no han sido eficaces, siendo uno de los grandes problemas a resolver en la elaboración de políticas públicas que expresen la voluntad política de dar solución al problema de la pobreza.

3. Ecuador no ha estado exento del impacto de la COVID-19, causando incrementos en el nivel pobreza. Las políticas públicas aplicadas por los gobiernos en los últimos 15 años anteriores al 2020, hoy se ven amenazadas quedando muchos retos que dependen de la voluntad gubernamental y, particularmente del trabajo colectivo de todos los actores sociales.

\section{REFERENCIAS}

Acosta, A. y. (2009). Siempre más democracia, nunca menos. A manera de prólogo. En D. Quirola, Sumak Kawsay: hacia un nuevo pacto social en armonía con la naturaleza. Quito Ecuador: Ediciones Abya Yala, Quito, Ecuador.

Asamblea Constituyente. (2008). Constitución de la República del Ecuador. Quito. 
Banco Mundial. (2020). Panorama general de la pobreza. Obtenido de https://www.bancomundial.org/es/topic/poverty/overview

Bazán Ojeda, A., \& Quintero Soto, M. L. (enero-junio de 2011). Evolución del concepto de pobreza y el enfoque multidimensional para su estudio. Quivera, 13(1), 207-2019.

Buen Vivir. (s.f.). Buen Vivir Plan Nacional 2013 - 2017. Recuperado el 20 de Junio de 2018, de Buen Vivir: https://www.unicef.org/ecuador/Plan_Nacional_Buen_Vivir_20132017.pdf

Comisión económica para América Latina y el Caribe. (2006). Guía de Asistencia técnica para la producción y el uso de indicadores de género. Guía de Asistencia técnica para la producción y el uso de indicadores de género. Santiago de Chile: CEPAL.

Comisión Económica para América Latina y el Caribe. (2006). Guia de asitencia técnica para la producción y el uso de indicadores de género. Santiago de Chile: CEPAL.

Constitución de la República del Ecuador. (2008). Recuperado el 15 de Junio de 2018, de https://www.oas.org/juridico/pdfs/mesicic4_ecu_const.pdf

Foro económico Mundial. (24 de 01 de 2015). http://noticias.starmedia.com/. Obtenido de http://noticias.starmedia.com/economia/resumen-completo-foro-economico-mundialdavos-2015.html

Galiano Maritan, G. y. (enero-junio de 2018). Análisis constitucional de los derechos personalísimos y su relación con los derechos del buen vivir en la Constitución de Ecuador". Revista deDerecho Privado. Universidad Externado de Colombia. (No. 34), 123-156.

Galiano Maritan, G., \& Tamayo Santana, G. (enero junio de 2018). Análisis constitucional de los derechos personalísimos y su relación con los derechos del buen vivir en la Constitución de Ecuador. Revista de Derecho Privado. Universidad Externado de Colombia (34), 123-156.

Girondella Mora, L. (2011). ¿Qué es pobreza relativa?

Hume, D. (2012). Understanding and explaining global proverty. Global proverty: how global governanve is failing the poor. Londres: Routledge. 
Hume, D. (3 de septiembre de 2012). Understanding and explaining global proverty. Global proverty: how global governanve is failing the poor.Londres: Routledge (Primera ed.). London: Routledge.

INEC. (2018). Reporte de pobreza y desigualdad. Quito: http://www.ecuadorencifras.gob.ec/documentos/web-inec/POBREZA/2018/Junio2018/Informe_pobreza_y_desigualdad-junio_2018.pdf.

León Segura, C. M. (mayo-agosto de 2016). Pobreza, medio ambiente y proactividad del Derecho. Revista Órbita Pedagógica, 3(2).

Martín Ravallion. (2015). Las línas de pobreza en la teoría y en la práctica. Banco Mundial.

Mero Figueroa, M., \& Alvarado-Gastiaburo, Á. C. (septiembre de 2018). Tendencias de la pobreza y la desigualdad en el Ecuador y América Latina. Revista Polo del Conocimiento, 3(24), 458-485.

Miniterio de Desarrollo Urbano. (2020). Más de 255.000 viviendas entregadas en seis años. Obtenido de https://www.habitatyvivienda.gob.ec/mas-de-255-000-viviendasentregadas-en-seis-anos/

Mora, L. G. (2011). ¿Qué espobreza relativa? ContraPeso.Info, 20-28.

Niemietz, K. (2011). A new understanding of poverty. Londres: The insitute of Economic Affairs.

Niemietz, K. (octubre de 2011). A New Understanding of Poverty: Poverty Measurement and Policy Implications. London: The Institute of Economic Affairs. 40(04), 857-858. doi:10.1017/S0047279411000407

ONU. (1995). Informe de desarrollo humano 1995. New York: Copenhague: Naciones Unidas. Organización de Naciones Unidas. (1995). Informe de desarrollo humano 1995. Copenhague: Naciones Unidas.

OXFAM Internacional. (2021). Los megas ricos han recuperado las pérdidas ocasionadas por la pandemia en un tiempo récord, mientras que miles de millones de personas viviránen situación de pobreza al menos una década. Obtenido de https://www.oxfam.org/es/notas-prensa/losmega-ricos-han-recuperado-las-perdidas- ocasionadas-por-la-pandemia-en-un-tiempo 
Pérez, P. G. (17 de 10 de 2018). https://definicion.de/pobreza/. Obtenido de https://www.google.com.ec/search?q=definici\%C3\%B3n+de+pobreza\&oq=definici\% $\mathrm{C} 3 \% \mathrm{~B} 3 \mathrm{n}+\mathrm{de}+$ pobreza+\&aqs $=$ chrome..69i57j015.6567j1j8\& sourceid $=$ chrome \&ie $=\mathrm{UT}$ F-8

Peumans, H. (1967). Valoración de proyectos de inversión. Recuperado el 18 de Junio de 2018 PNUD. (2015). Informe sobre desarrollo humano.

PNUD. (2015).

Informe sobre Desarrollo Humano. dr.undp.org/sites/default/files/2015_human_development_report_overview_-_es.pdf.

Primicias.ec. (2021). Pobres necesitan una década para recuperarse, los ricos ya lo hicieron. . Obtenido de https://www.primicias.ec/noticias/economia/pobres-necesitan-decadapara-recuperarse-ricos-ya-hicieron-covid/

Ravallion, M. (2015). Las líneas de pobreza en la teoría y en la práctica. Cuarto Taller Regional sobre la medición de la pobreza: El método de las líneas de pobreza, Banco Mundial, 113-143.

Secretaría Nacional de Planificación y Desarrollo - Senplades, 2. (2017). Plan Nacional para el Buen Vivir 2017-2021.

Sen, A. (2001). Capacidad y bienestar. Obtenido de http:// www.eumed.net/cursecon/textos/SEn-capacidad_y bienestar.htm.

Sen, A. (2002). El desarrollo como libertad. Roma: Universidad Torcuato di Tella.

SENPLADES. (2008). Plan Nacional del Buen Vivir. Quito.

SIISE. (2018). Líneas de pobreza y extrema pobreza de consumo. Quito: http://www.siise.gob.ec/siiseweb/PageWebs/POBREZA/ficpob_P39.htm. 